Supplement of Atmos. Chem. Phys., 19, 13647-13679, 2019

https://doi.org/10.5194/acp-19-13647-2019-supplement

(c) Author(s) 2019. This work is distributed under

the Creative Commons Attribution 4.0 License.

(c) (1)

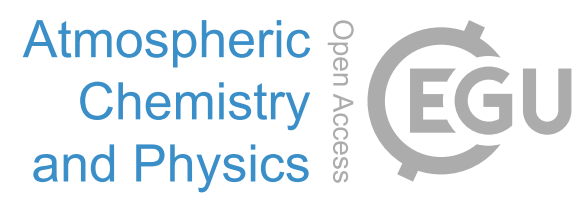

Supplement of

\title{
Technical note: Reanalysis of Aura MLS chemical observations
}

Quentin Errera et al.

Correspondence to: Quentin Errera (quentin.errera@aeronomie.be)

The copyright of individual parts of the supplement might differ from the CC BY 4.0 License. 


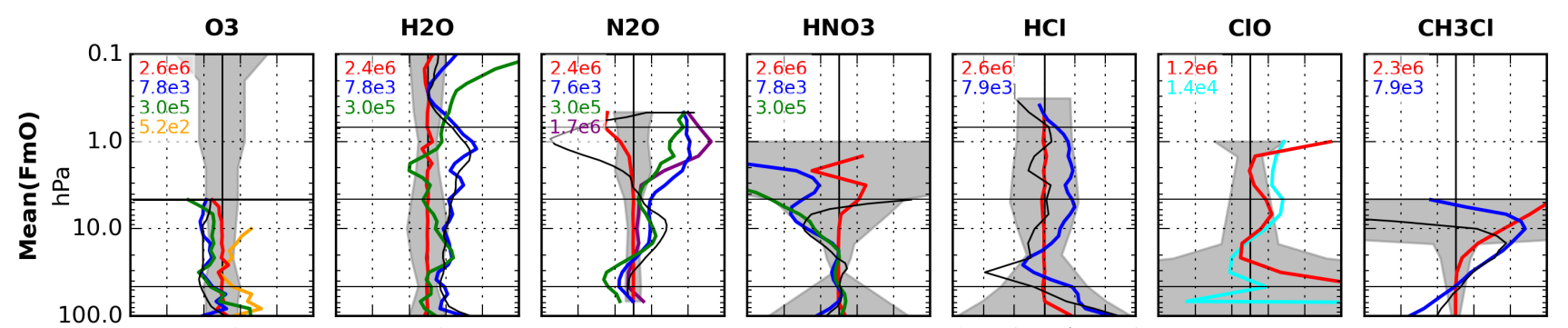

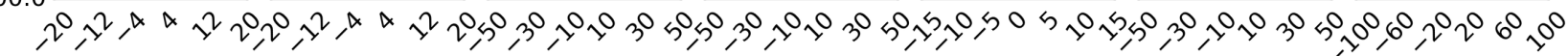
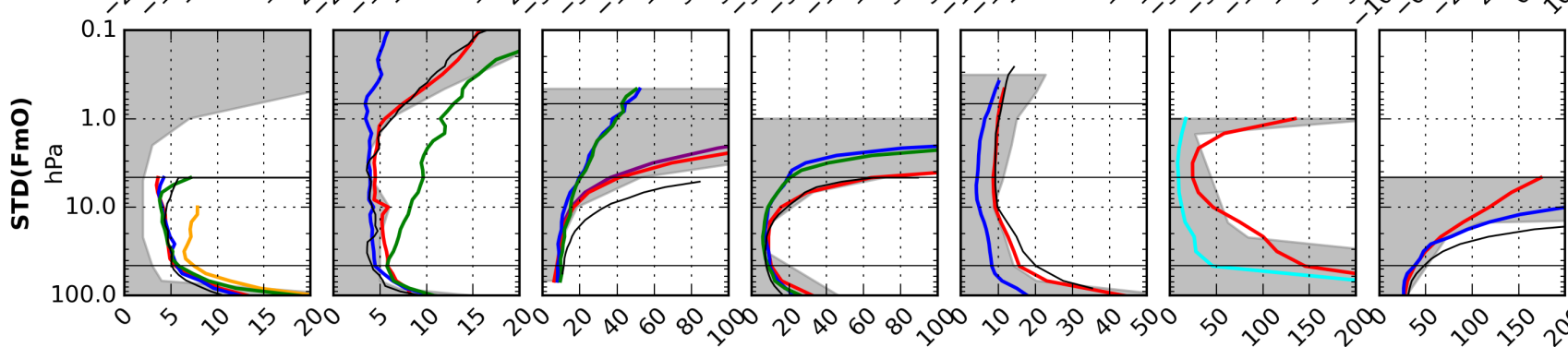

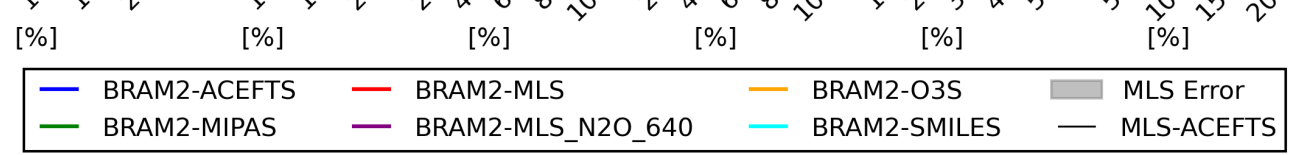

Figure S1. As Fig. 5 but between $60^{\circ} \mathrm{S}-30^{\circ} \mathrm{S}$.
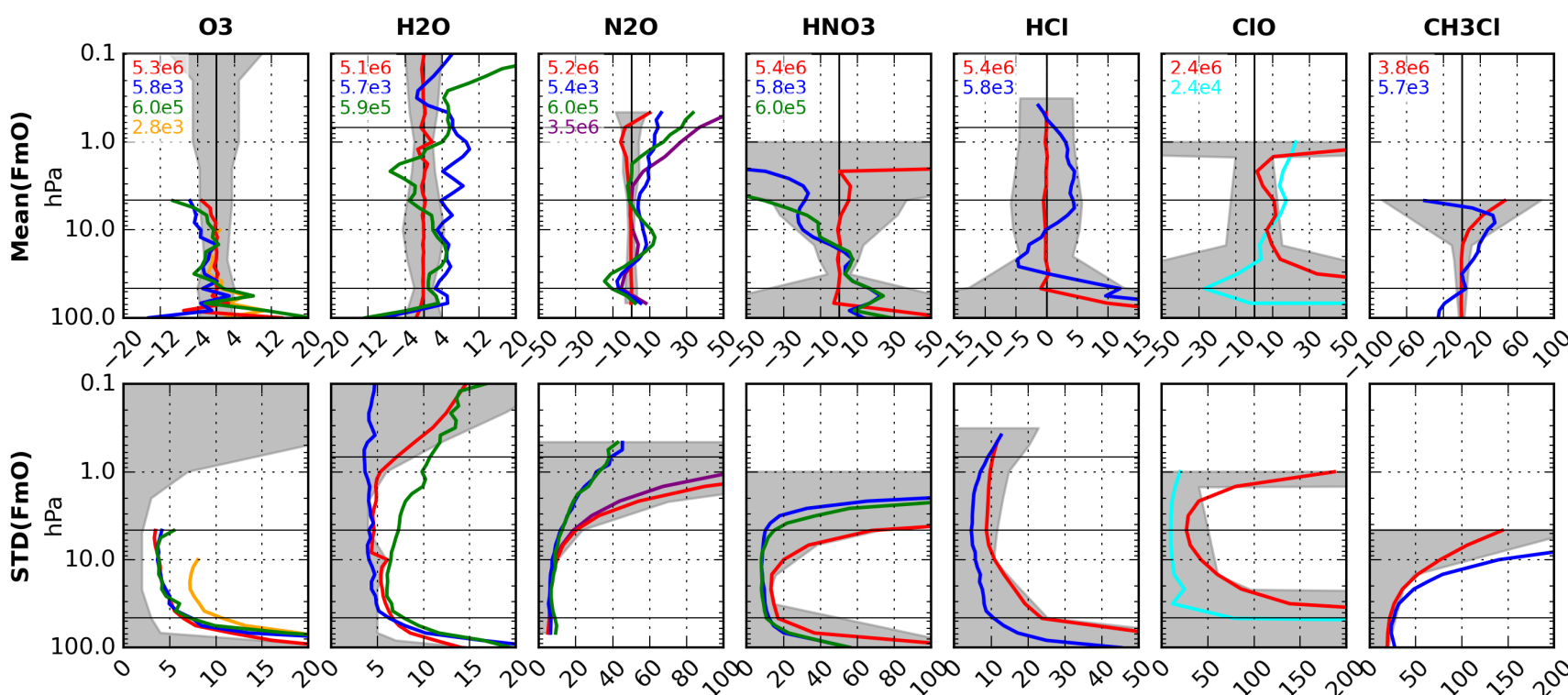

[\%]

[\%]

[\%]

[\%]

[\%]
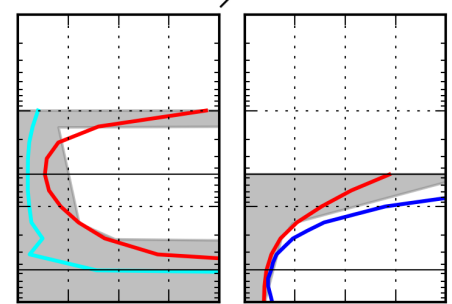

- BRAM2-ACEFTS - BRAM2-MLS

BRAM2-MLS_N2O_640

- BRAM2-O3S

BRAM2-SMILES

[\%]

[\%]

Figure S2. As Fig. 5 but between $30^{\circ} \mathrm{S}-30^{\circ} \mathrm{N}$. 


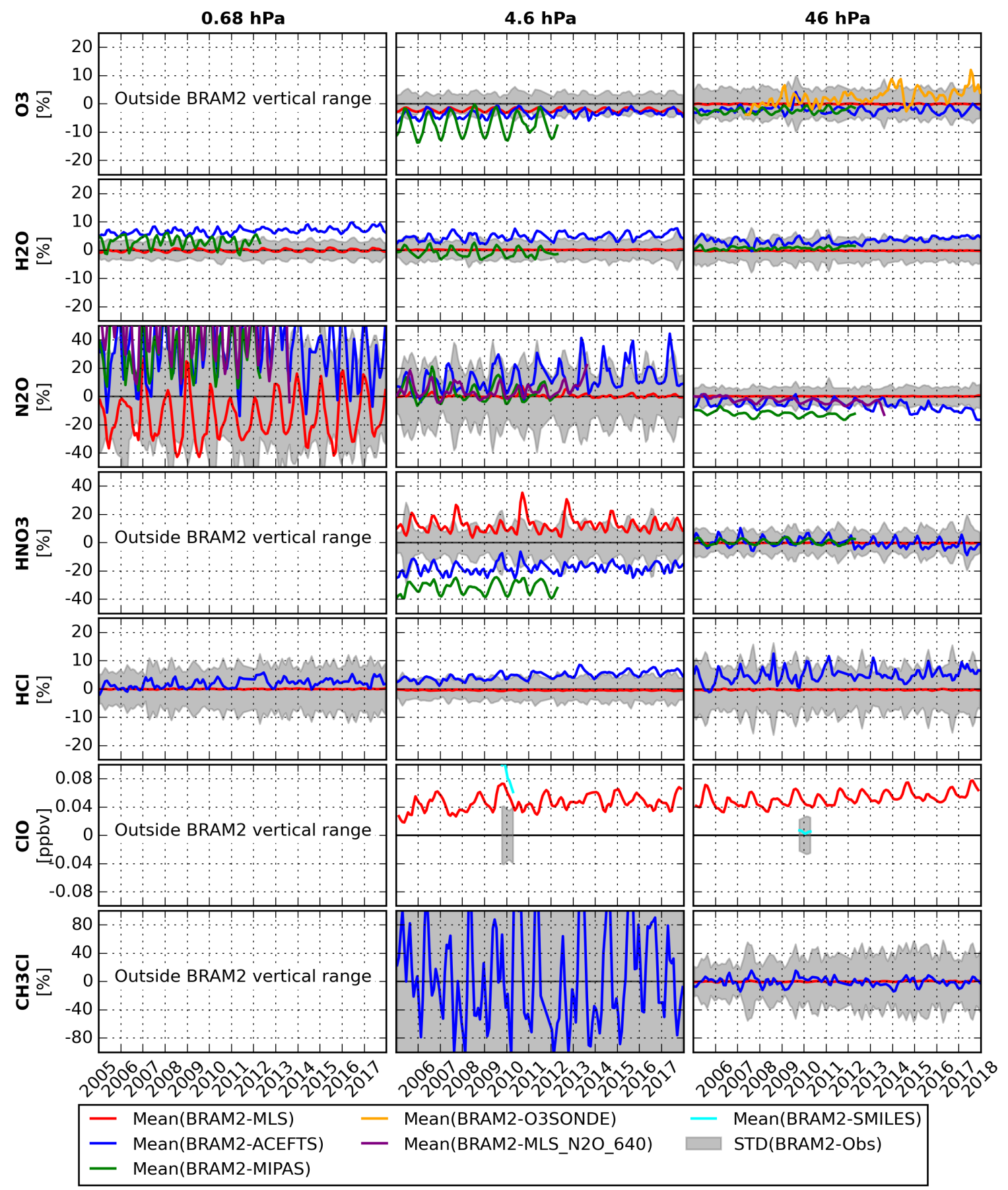

Figure S3. As Fig. 6 but between $60^{\circ} \mathrm{S}-30^{\circ} \mathrm{S}$. 


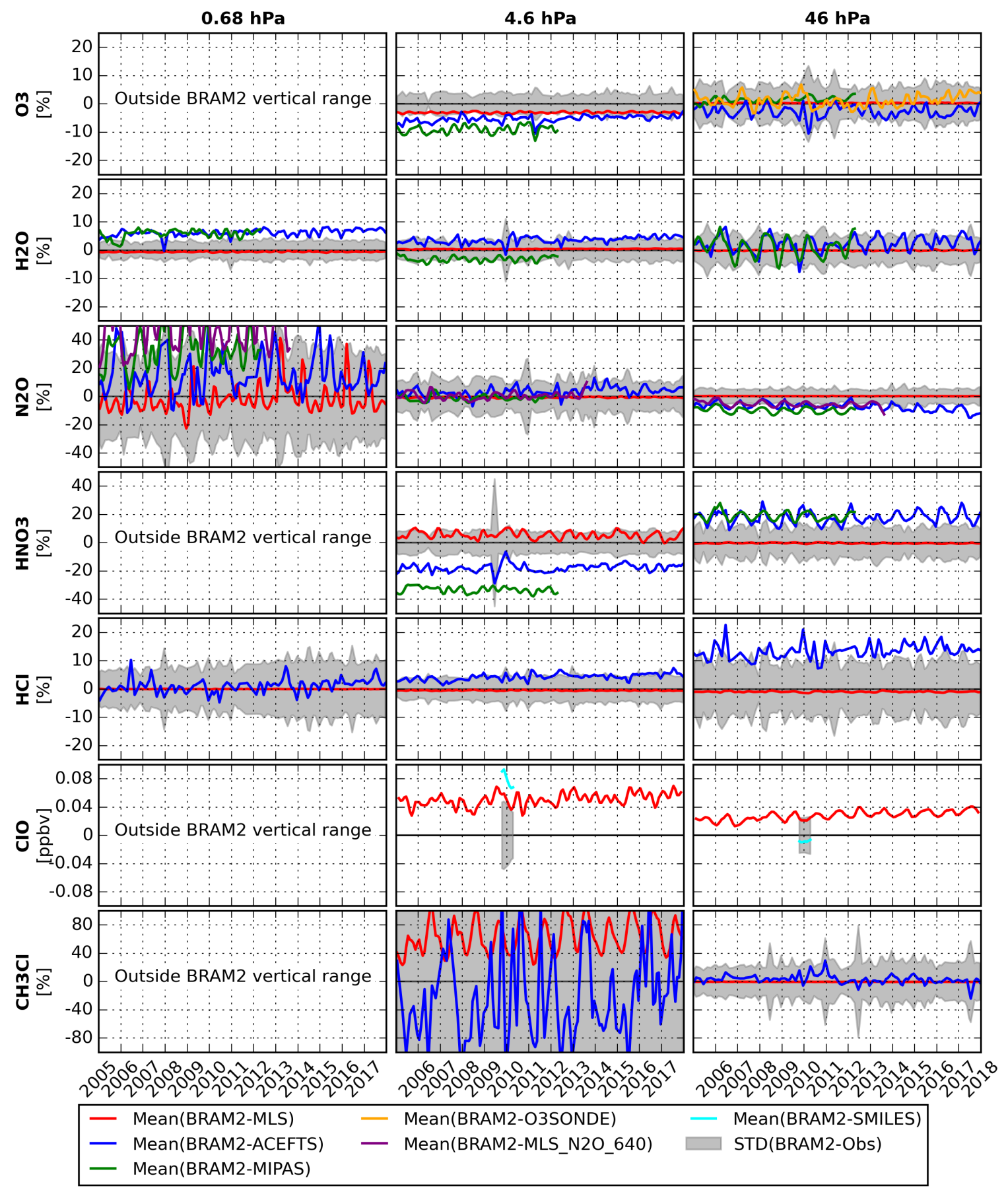

Figure S4. As Fig. 6 but between $30^{\circ} \mathrm{S}-30^{\circ} \mathrm{N}$. 


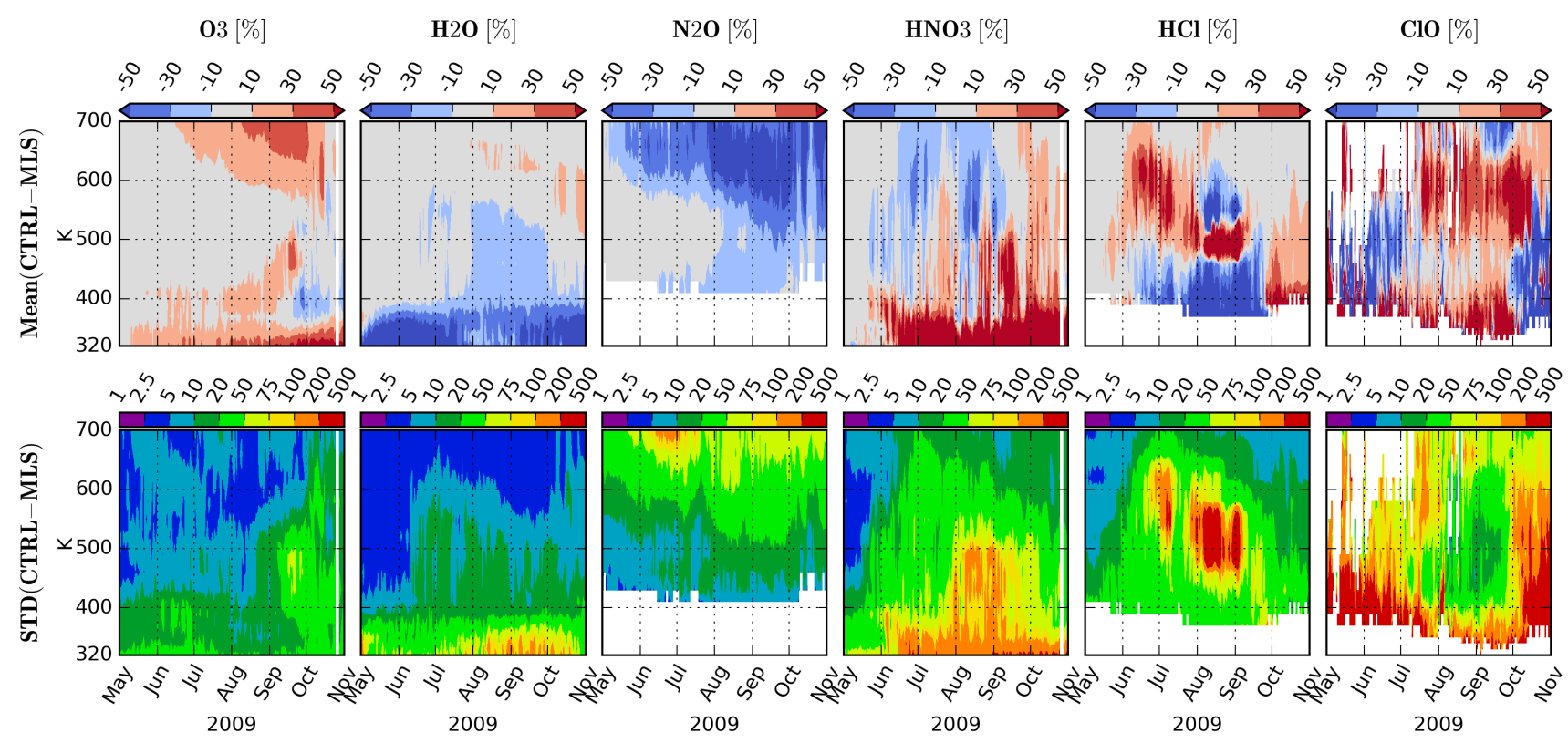

Figure S5. Mean (top) and standard deviations (bottom) of the difference (CTRL-MLS) for the conditions shown in Fig. 7. 


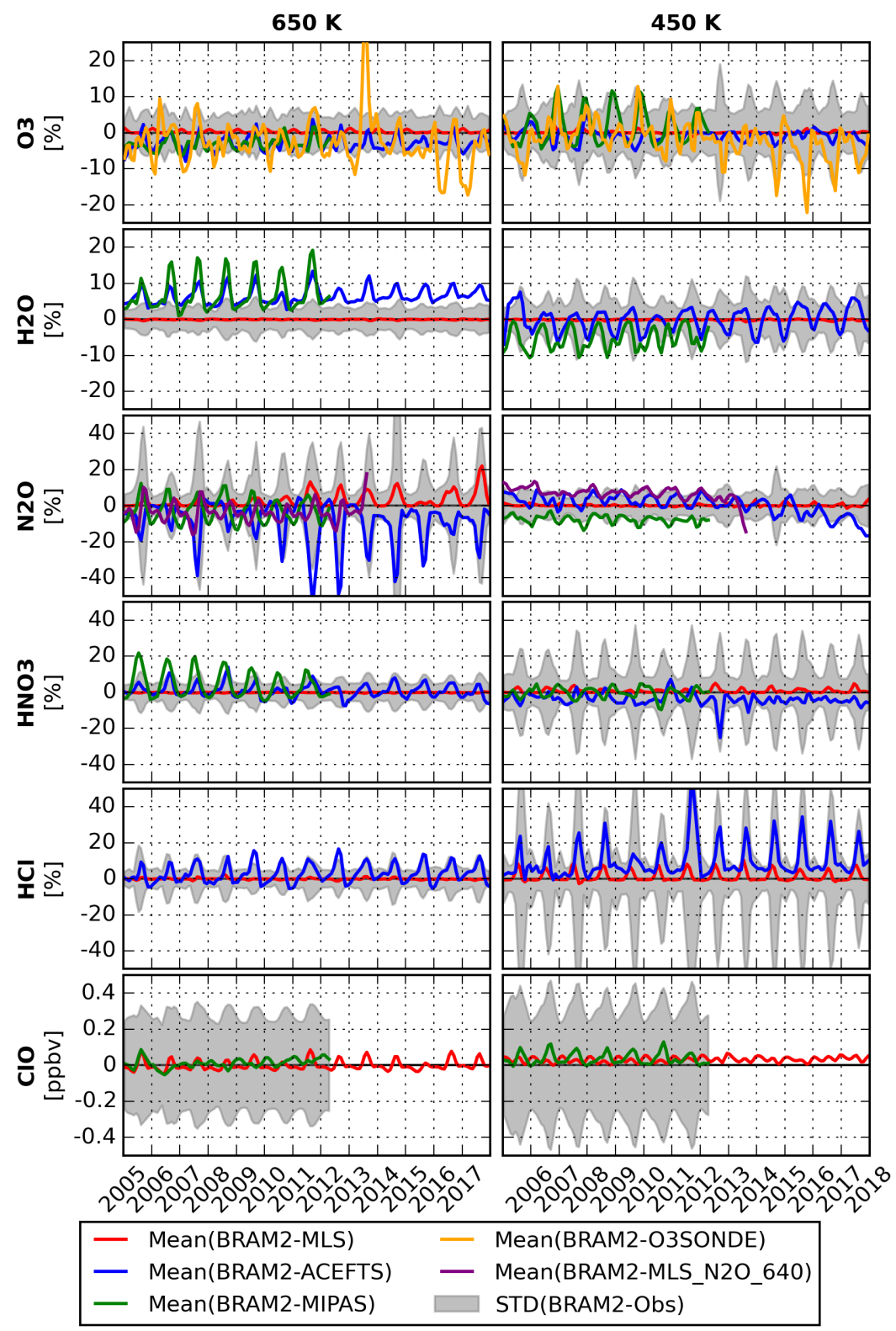

Figure S6. As Fig. 8 but in the outer Antarctic polar vortex between $\left(75^{\circ} \mathrm{S}-60^{\circ} \mathrm{S}\right.$ of equivalent latitude). 


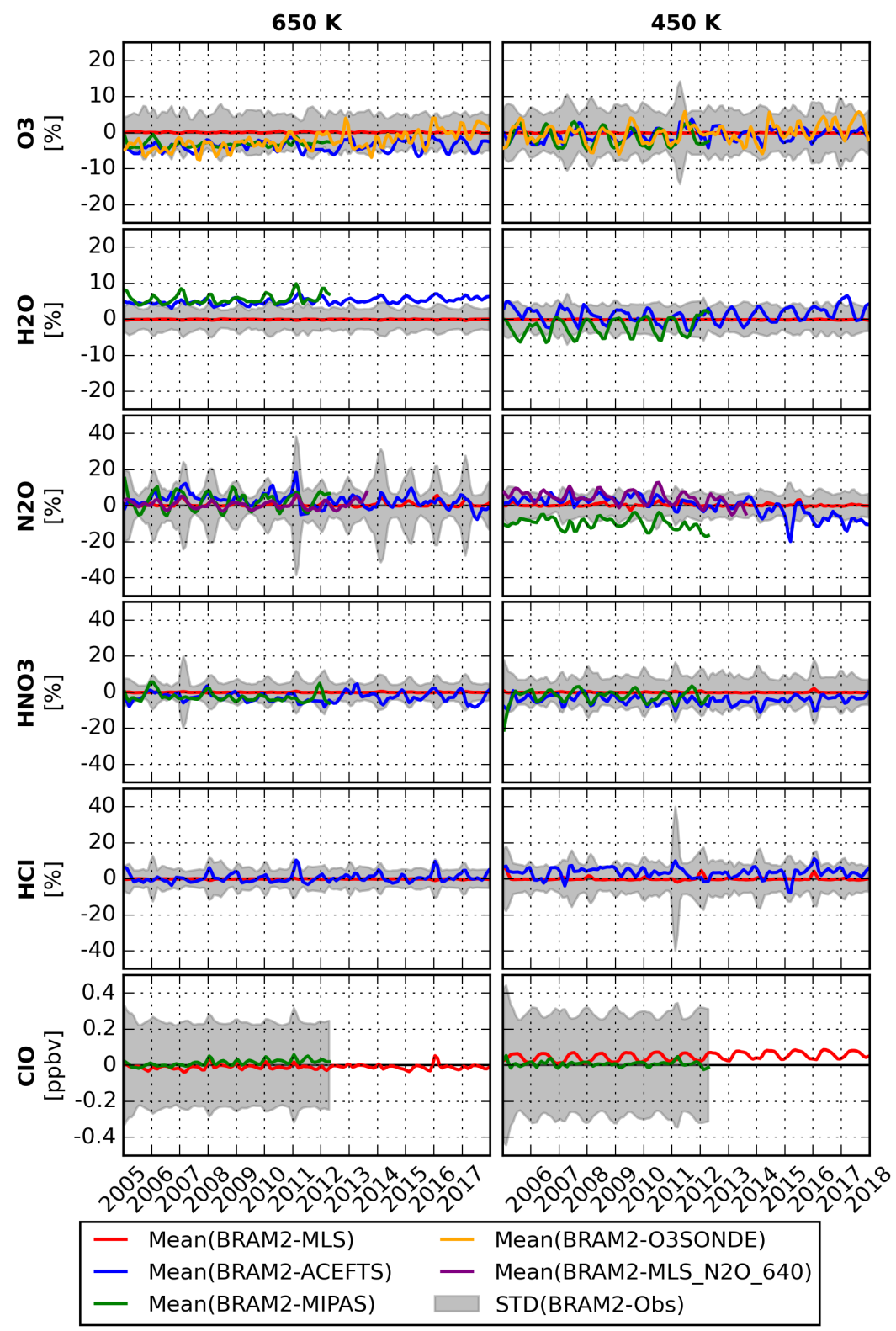

Figure S7. As Fig. 8 but in the outer Arctic polar vortex between $\left(60^{\circ} \mathrm{N}-75^{\circ} \mathrm{N}\right.$ of equivalent latitude). 


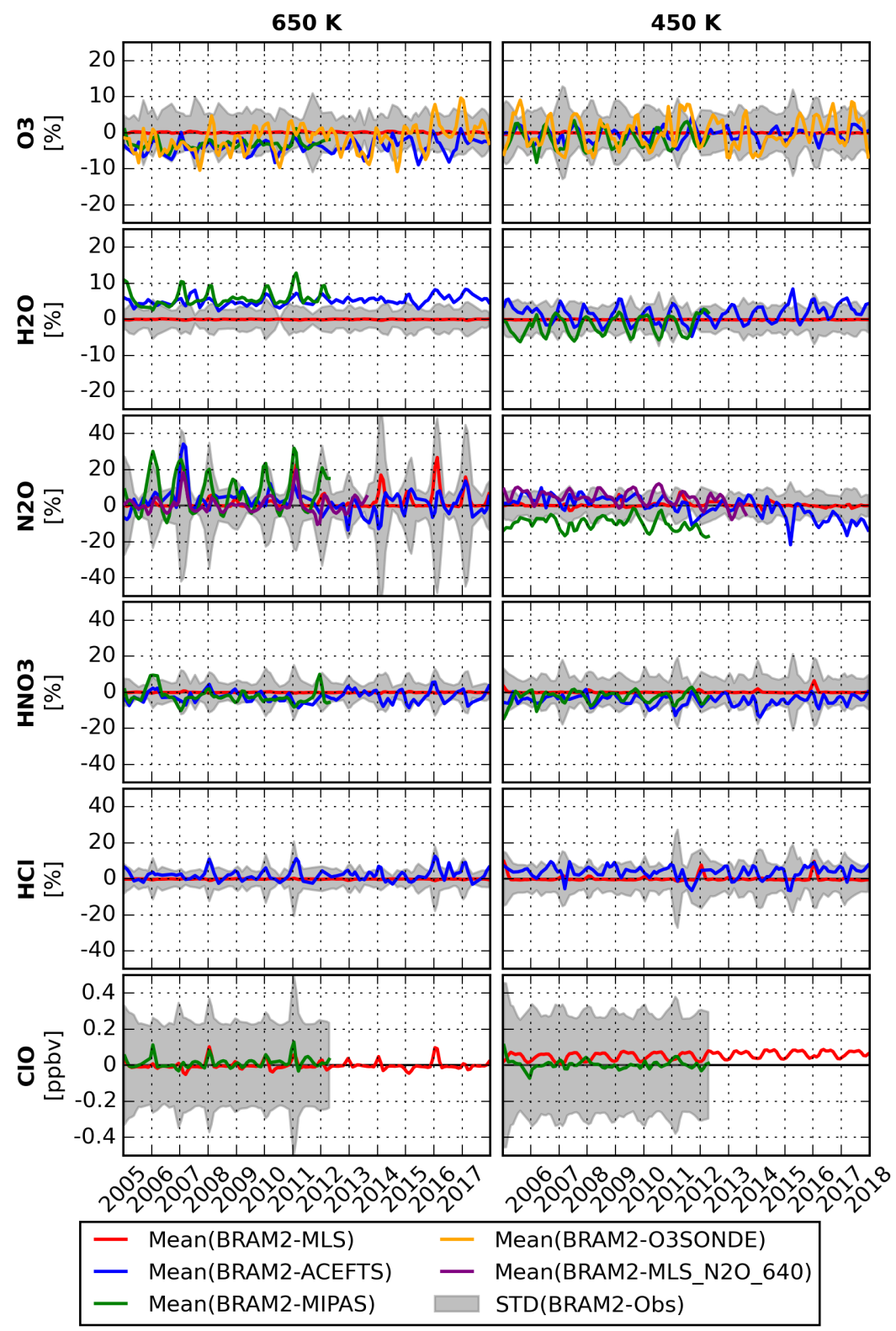

Figure S8. As Fig. 8 but in the outer Arctic polar vortex between $\left(75^{\circ} \mathrm{N}-90^{\circ} \mathrm{N}\right.$ of equivalent latitude). 

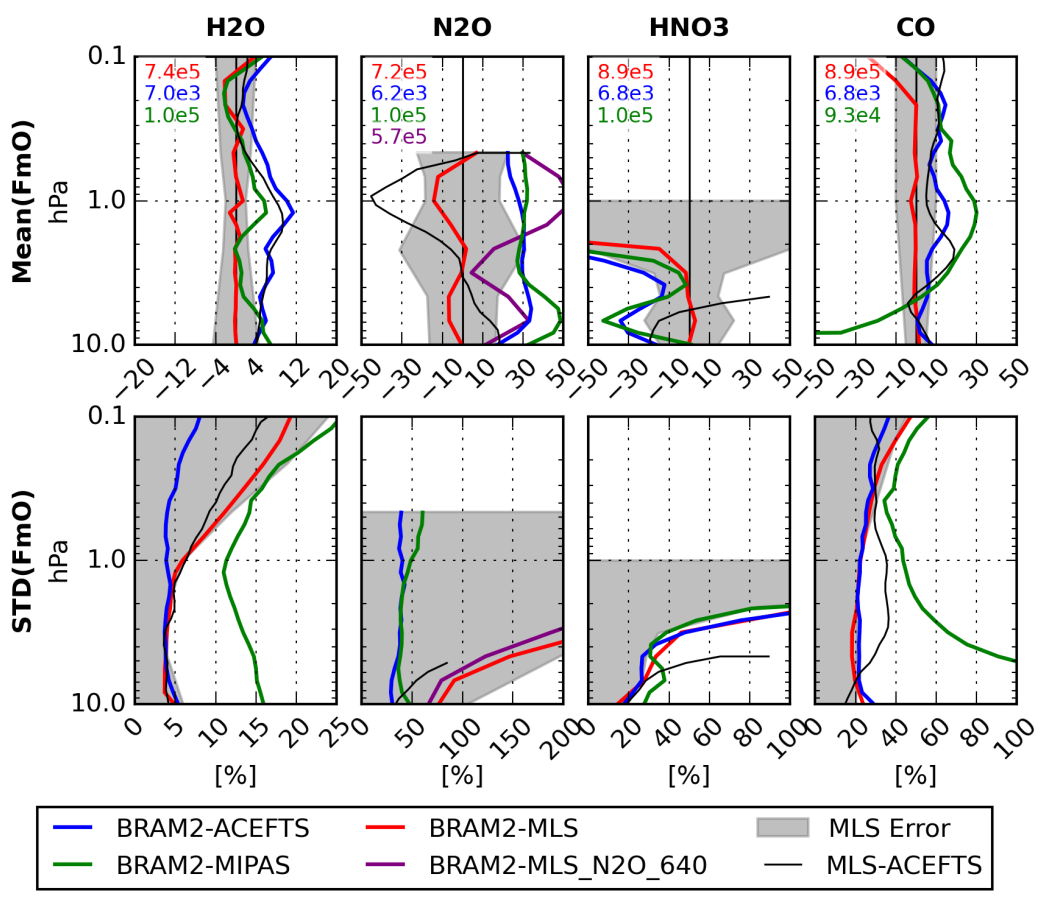

Figure S9. As Fig. 10 but between $90^{\circ} \mathrm{S}-60^{\circ} \mathrm{S}$. 

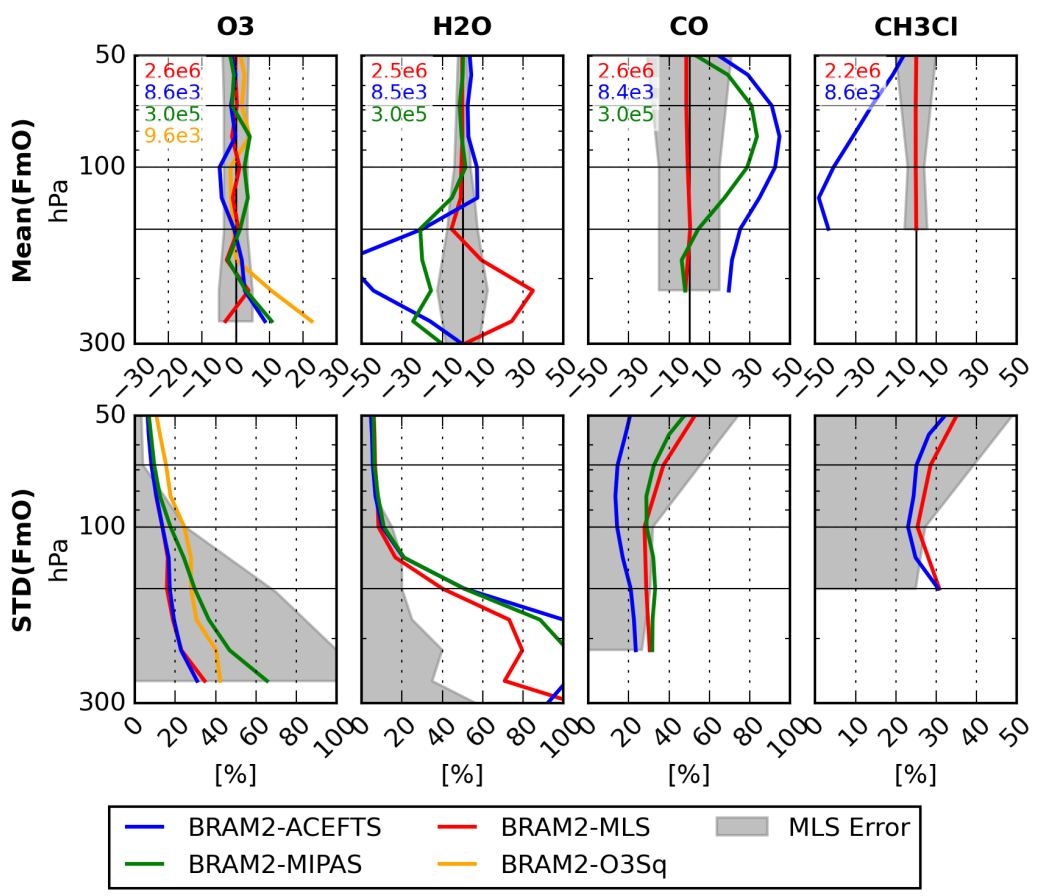

Figure S10. As Fig. 12 but between $30^{\circ} \mathrm{N}-60^{\circ} \mathrm{N}$.
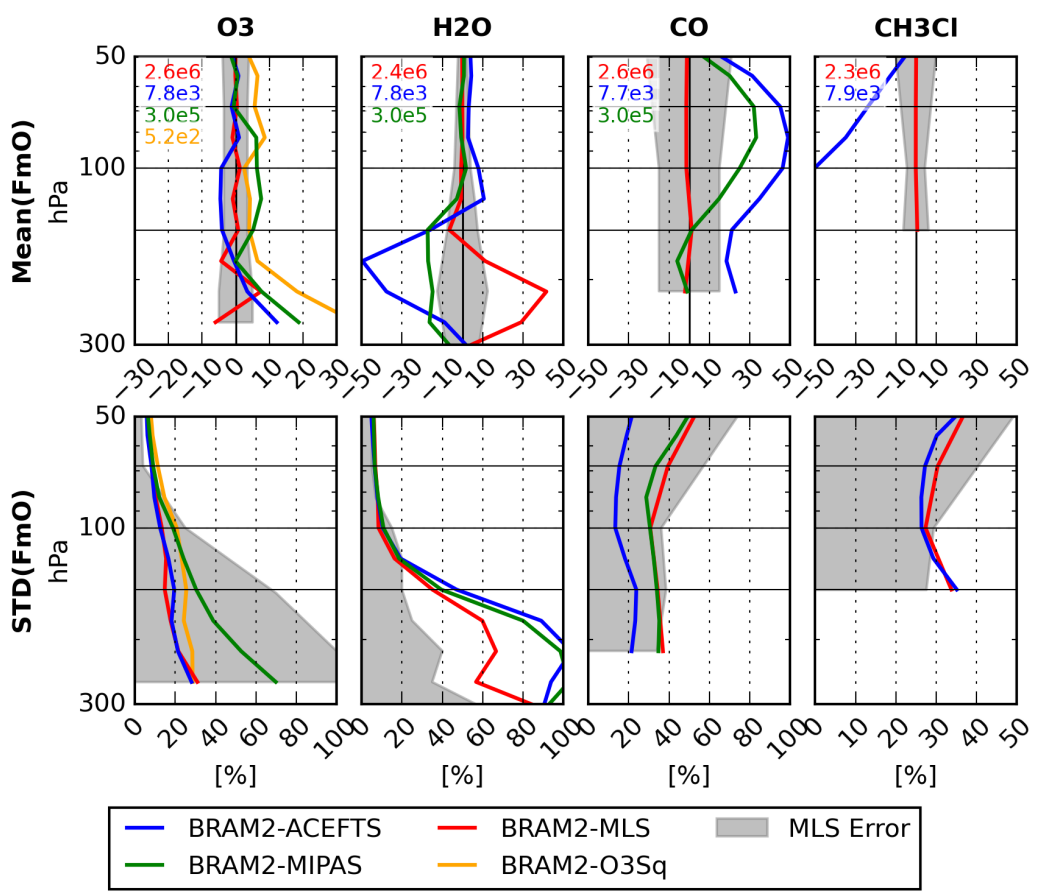

Figure S11. As Fig. 12 but between $60^{\circ} \mathrm{S}-30^{\circ} \mathrm{S}$. 


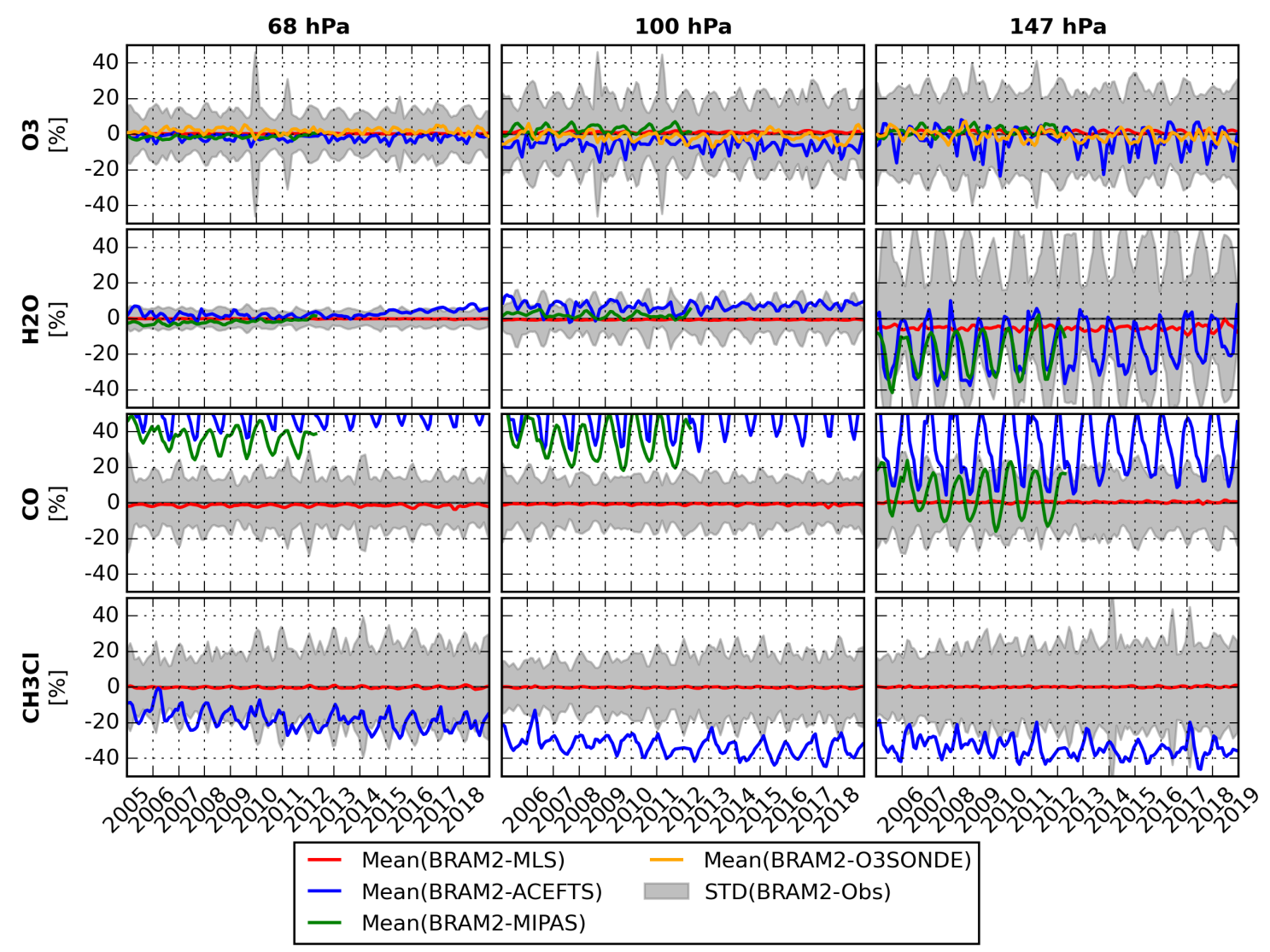

Figure S12. As Fig. 12 but between $30^{\circ} \mathrm{N}-60^{\circ} \mathrm{N}$. 


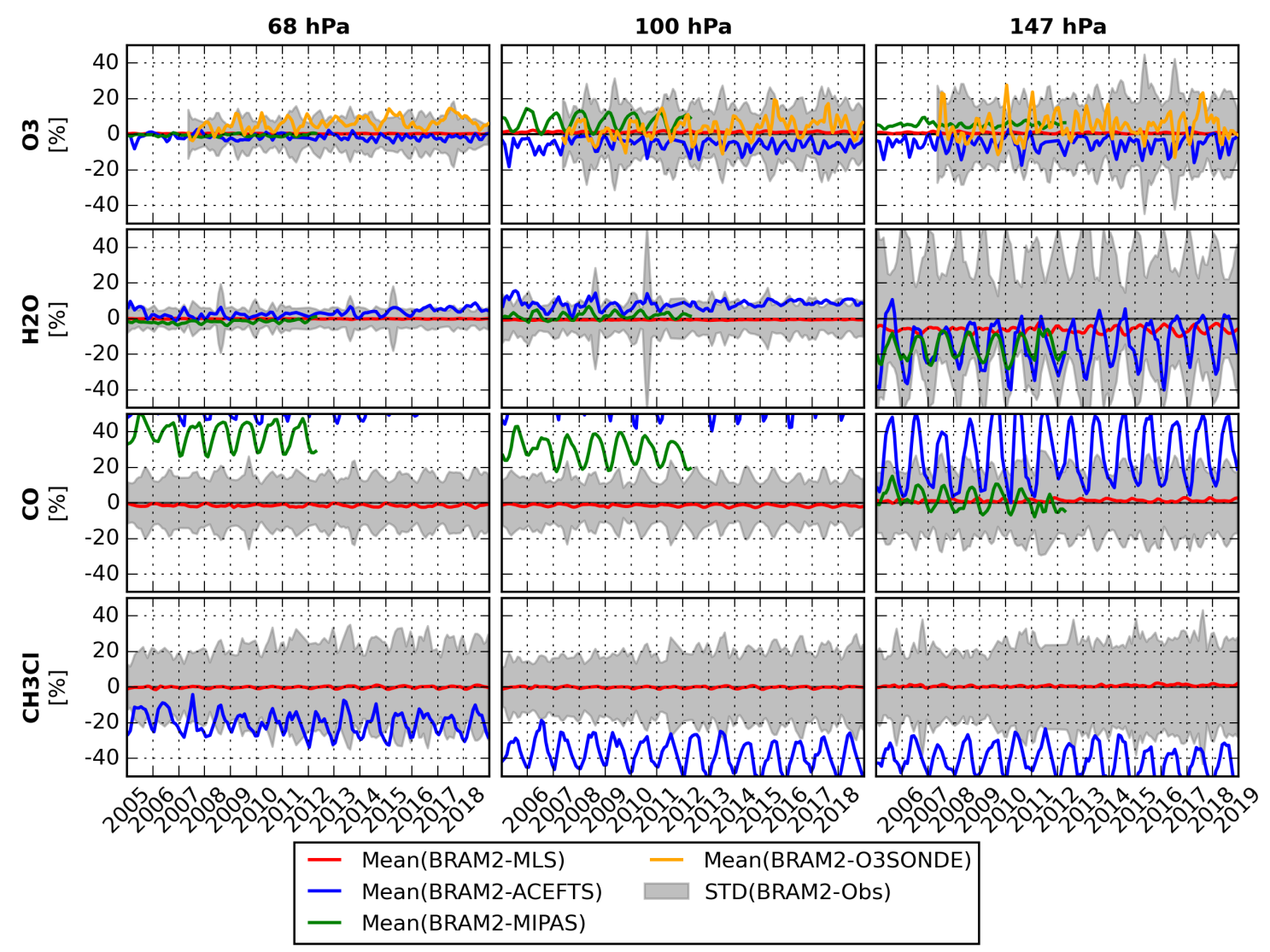

Figure S13. As Fig. 13 but between $60^{\circ} \mathrm{S}-30^{\circ} \mathrm{S}$. 
Table S1. Mean difference and standard deviation between BRAM2 and observations for the 2005-2017 period at three typical levels in the ex-UTLS: 68, 100 and $147 \mathrm{hPa}$. Values are in \%, normalized by BRAM2. Abbreviations: Not Assimilated (N. A.).

\begin{tabular}{l|l|c|c|c}
\hline Species & Instruments & $68 \mathrm{hPa}$ & $100 \mathrm{hPa}$ & $147 \mathrm{hPa}$ \\
\hline $\mathrm{O}_{3}$ & MLS & $0 \pm 8 \%$ & $1 \pm 13 \%$ & $1 \pm 16 \%$ \\
& ACE-FTS & $-2 \pm 8 \%$ & $-5 \pm 13 \%$ & $-1 \pm 17 \%$ \\
& MIPAS & $-1 \pm 9 \%$ & $4 \pm 17 \%$ & $1 \pm 30 \%$ \\
& O3sondes & $2 \pm 15 \%$ & $-2 \pm 25 \%$ & $-1 \pm 28 \%$ \\
\hline $\mathrm{H}_{2} \mathrm{O}$ & MLS & $0 \pm 7 \%$ & $-1 \pm 8 \%$ & $-5 \pm 40 \%$ \\
& ACE-FTS & $2 \pm 6 \%$ & $7 \pm 10 \%$ & $-20 \pm 50 \%$ \\
& MIPAS & $-2 \pm 6 \%$ & $1 \pm 11 \%$ & $-20 \pm 50 \%$ \\
\hline $\mathrm{CO}$ & MLS & $-1 \pm 35 \%$ & $-1 \pm 28 \%$ & $0 \pm 29 \%$ \\
& ACE-FTS & $40 \pm 14 \%$ & $42 \pm 14 \%$ & $25 \pm 22 \%$ \\
& MIPAS & $30 \pm 33 \%$ & $30 \pm 28 \%$ & $5 \pm 33 \%$ \\
\hline $\mathrm{CH}_{3} \mathrm{Cl}$ & MLS & $0 \pm 30 \%$ & $0 \pm 25 \%$ & $0 \pm 30 \%$ \\
& ACE-FTS & $-20 \pm 25 \%$ & $-40 \pm 23 \%$ & $-45 \pm 30 \%$ \\
\hline
\end{tabular}

